\title{
Scenario selection of wind forecast errors for stochastic unit commitment: A UK case study
}

Link to publication record in Manchester Research Explorer

\section{Citation for published version (APA):}

Ochoa, L. F., Gomez, J., \& Ortega-Vazquez, M. (2014). Scenario selection of wind forecast errors for stochastic unit commitment: A UK case study. In IEEE/PES Innovative Smart Grid Technologies ISGT Europe 2014 (pp. 1-6)

\section{Published in:}

IEEE/PES Innovative Smart Grid Technologies ISGT Europe 2014

\section{Citing this paper}

Please note that where the full-text provided on Manchester Research Explorer is the Author Accepted Manuscript or Proof version this may differ from the final Published version. If citing, it is advised that you check and use the publisher's definitive version.

\section{General rights}

Copyright and moral rights for the publications made accessible in the Research Explorer are retained by the authors and/or other copyright owners and it is a condition of accessing publications that users recognise and abide by the legal requirements associated with these rights.

\section{Takedown policy}

If you believe that this document breaches copyright please refer to the University of Manchester's Takedown Procedures [http://man.ac.uk/04Y6Bo] or contact uml.scholarlycommunications@manchester.ac.uk providing relevant details, so we can investigate your claim.

\section{OPEN ACCESS}




\section{Scenario Selection of Wind Forecast Errors for Stochastic Unit Commitment: A UK case study.}

\author{
Jonathan Gomez-Martinez \\ The University of Manchester \\ Manchester M13 9PL, UK \\ jonathan.gomezmartinez@manchester.ac.uk
}

\author{
Miguel Ortega-Vazquez \\ The University of Washington \\ Seattle, WA 98195 \\ maov@uw.edu
}

\author{
Luis F Ochoa \\ The University of Manchester \\ Manchester M13 9PL, UK \\ luis_ochoa@ieee.org
}

\begin{abstract}
Integration of renewable generation is crucial in future generation mix of power systems as many are the benefits that it offers. At certain penetration levels, latent risks could materialize as a result of not adequately managing the additional variability, therefore this situation prompts the necessity of enhancing traditional tools to operate power systems. In this paper a sensitivity analysis is conducted to assess key variables that improve the performance of Stochastic Unit Commitment (SUC). Focus is given to the right number of scenarios that sufficiently capture the variability of wind power forecast errors, in which a trade-off between quality of solution and computation time is considered. The proposed methodology is tested with real data from the Balancing Mechanism Reporting System aiming to represent the UK Power System.
\end{abstract}

Keywords-Wind forecast error, stochastic unit commitment, power generation dispatch, scenario generation, scenario reduction.

\section{INTRODUCTION}

Low production cost and environmental benefits brought by wind power generation is the most important driving force behind its increasing role in generation capacity around the world. Nonetheless, the corresponding (forecast) uncertainties associated with this variable resource could lead to unexpected power balancing issues and trigger serious risks to system reliability. Without an assessment of these eventualities, system operators would have limited information on the likelihood and magnitude of possible hazards. Generally speaking, improvements in forecasting techniques will reduce the overall cost of the system. According to wind integration studies, some practices have to be considered: i) A long enough data base to anticipate rare events, ii) Increase the periodicity of updating information, and iii) Include the forecast error in operational planning tools [1-3].

As any weather driven power source, wind power lacks predictability and its effects on the deployment of resources to accommodate these vagaries are more acute as larger volumes are integrated to power systems. Therefore, definition of reserves is fundamental to absorb deviations between forecast and actual values in a cost-effective manner. In a first approach, deterministic models apply statistical analysis of the net demand to assign a fix value of reserve (3- $\sigma$ Method)[4]. In the UK system, National Grid assigns reserves as a percentage of the most recent foresight, for instance; if wind production is greater than $1500 \mathrm{MW}$, the amount of reserve should be $10 \%$ of the corresponding prediction in the next half hour[5]. In a more sophisticated outlook, Stochastic Unit Commitment dynamically assigns reserves according to the random parameter that is represented through the set of scenarios (e.g. load demand, wind power). One of the most successful implementations of Stochastic Programming in UC is WILMAR project, in which the adopted tree structure was sized to accomplish degrees of freedom but mainly for practical reasons due to calculation time[6,7]. In the case of scenario-based UC similar situation takes place: Qadrdan et al.[8] presents a probabilistic forecast to generate 1000 realizations of wind power and then reduced to 5 representative scenarios, partly explained by the complexity of solving gas and electricity network in the same formulation. Zhang et al.[9] proposes conditional forecast error that makes use of copula theory to perform probabilistic analysis in a scenario-based UC, in which 3000 scenarios were trimmed down to a computationally feasible number of 20. Morales et al.[10] developed a detailed scheme to allocate spinning and nonspinning reserves in systems with high wind penetration, taking into account network constraints. In the first phase of that proposal [10], 3018 scenarios were created and then narrowed down to 20 scenarios.

One question that needs to be asked, however, is whether the maximum feasible number of scenarios leads to acceptable solutions in the decision model (SUC). In some circumstances that number might underestimate the effect of the random parameter (under specification of the problem [11, 12]), whereas in other case it might unnecessarily increase computational burden. In Stochastic Programming this tradeoff is essential in order to have adequate solutions and reduce computation burden. In this paper, the scenario generation \& reduction method were tested together with the decision model (SUC)[13]. During the process of appraising both techniques together, it was found that the number of scenarios to characterize the random parameter (wind power forecast error) does not necessarily results in a larger number of scenarios. This conclusion is supported by the solution obtained in the decision model, owing to there is a saturation point at which more scenarios does not significantly increase the precision in the objective function. Advantages and disadvantages of this methodology are discussed in subsequent sections. The organization of the paper is as follows. Section II provides a statistical analysis of wind power forecast error in the UK system. Section III explains the scenario generation \& reduction method used in this work. Section IV describes the objective function and constrains of Scenario-based Unit 
Commitment. Section V demonstrates the usefulness of the methodology with real data aiming to mimic the UK power system, and Section VI completes the paper with pertinent conclusion.

\section{WIND POWER FORECAST ERROR}

\section{A. Main Assumptions}

According to recent figures reported by National Grid (NG), the market share of wind power in the UK is around $10 \%$ with an installed capacity of $9 \mathrm{GW}$ and it will increase to $45 \mathrm{GW}$ by 2020 , with offshore wind leading these technologies[14]. Therefore, the architecture of forecasting tools used by NG is constantly evolving. Presently, that engine represents a second generation that embodies physical, statistical and neural network models. Initially, the forecasting system predicts wind speed and then it transforms that information into wind power through wind power curves. Furthermore, different predictions are prepared in different windows for specific needs of the operational planning process. Typically, weekly programs are meant to buy fuels and manage hydro reservoirs ( 7 days Ahead), then daily programs determine generation scheduling for next $24 \mathrm{hrs}$ and finally generation re-schedule is performed every 4 hours in case unexpected deviations occur [15]. This information is publically available in the website of the Balancing Mechanism Reporting System of the UK [16], and correspond to the aggregated production of all wind farms that are metered by National Grid. For this research, the available data base comprises twenty three months with sampling interval of one hour resolution, from January 2012 until November 2013. Hereinafter the term forecast error $\left(e_{t}\right)$ will be defined as a difference between wind forecast and wind capability (actual wind production injected to the network plus wind curtailment) (1). The inclusion of wind curtailment $(w c)$ is based on the fact that forecast is created from wind speed regardless any further reduction due to balancing purposes. After recording deviations of twenty three months data, the largest dispersion of error's magnitude was found in the forecast that is prepared 7 days ahead (e7DAF), whose standard deviation is $1136 \mathrm{MW}$. Whereas deviations in 1 day ahead and $4 \mathrm{hrs}$ ahead were smaller(367MW and 304MW respectively).

$$
e_{t}=x_{\text {forecast }, t}-\left(x_{\text {actual }}+x_{\text {curtailment }, t}\right)
$$

\section{B. Properties of wind power forecast error}

During the process of analyzing the data base, a positive correlation was found between the forecast error and the forecast of wind power. Thus, the larger the predicted value of wind power, the larger is the forecast error (see Figure 1). Furthermore, the forecast that is closer to real time ( 1 day and $4 \mathrm{hr}$ ahead) exhibited a particular characteristic of having most of the error's magnitude in a range between 0 and $20 \%$ of the registered capacity, see Figure 2.

\section{SCENARIO GENERATION \& REDUCTION}

\section{A. Percentage Time Series}

In a first attempt to reproduce deviations between forecast and actual values of wind power, a modified version of percentage time series is used[17];

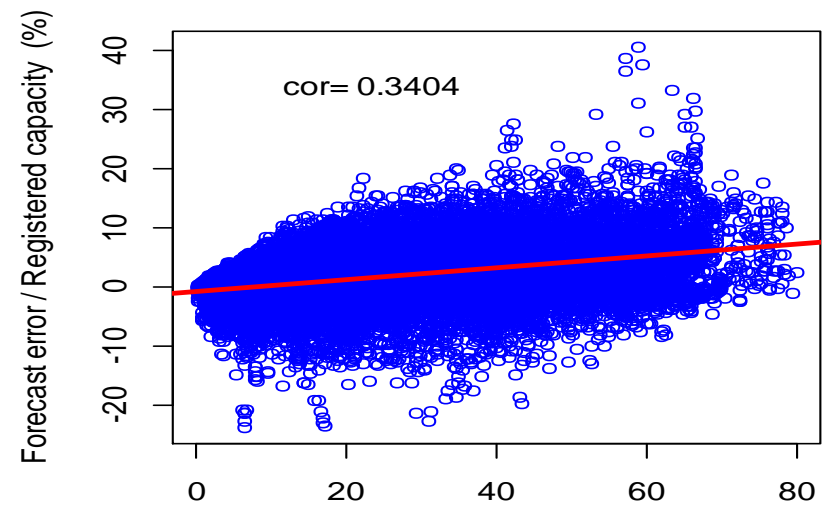

Predicted wind power / Registered capacity (\%)

Fig. 1. Correlation between forecast error and predicted wind power in one day ahead.

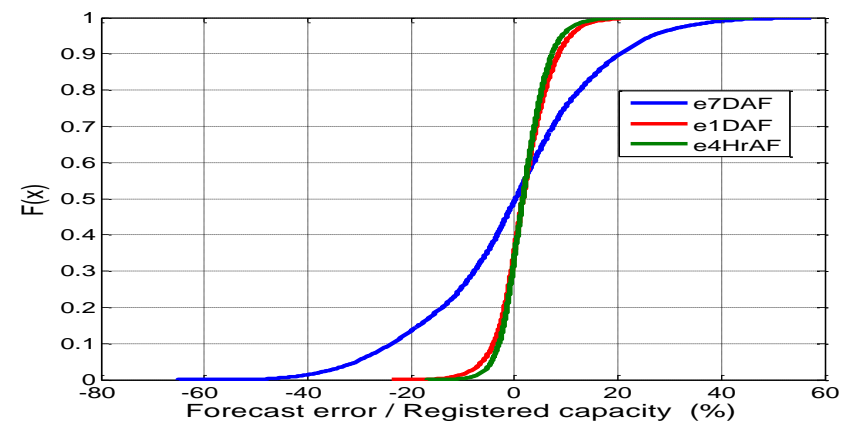

Fig. 2. Cumulative relative frequency of forecast error.

Initially, forecast errors are calculated according to equation (1) for the database that was described in previous section. Then, these errors are normalized according to the capacity registered by National Grid[16]. Next, the Box \& Jenkins methodology is applied to identify, estimate and validate a model for each forecast error horizon[18]. Lastly, each model reproduces 1000 realizations of forecast error taking into account their inter-temporal correlation (Figure 3).

\section{B. Scenario Reduction}

Dimensionality is one of the main drawbacks for stochastic programming, thus reduction techniques becomes essential to narrow down the number of scenarios whilst statistical properties of the random parameters are retained. In this work a heuristic called backward deletion is implemented according to Barth et al. [19]. First of all, a Euclidean distance $d\left(\xi^{(i)}, \xi^{(j)}\right)$ is calculated between all scenario pairs, according to equation (2). These information is stored in a distance matrix that has the same dimension as the initial number of scenarios (e.g. $n_{s}=1000$ ). Hence, each element in the matrix contains the Euclidean distance between scenario $i$ and scenario $j$.

$$
\begin{gathered}
d\left(\xi^{(i)}, \xi^{(j)}\right)=\sqrt{\sum_{s=1}^{n_{T}}\left(p_{s}^{(i)}-p_{s}^{(j)}\right)^{2}} \\
\pi^{\left(s^{*}\right)} \min _{s \neq s^{*}} d\left(\xi^{(s)}, \xi^{\left(s^{*}\right)}\right) \\
\pi^{(s)}=\pi^{(s)}+\pi^{\left(s^{*}\right)}
\end{gathered}
$$




\section{Accepted Paper}

This method assumes that scenarios with smaller distance are similar therefore it is doable to remove one of them. Moreover, the probability of each scenario $(\pi)$ is pondered along with the Euclidean distance, owing to unlikely scenarios are prone to be delated (3). Once the decision is made regarding the scenario that is deleted, the next step is to update the probability of the nearest scenario $\pi^{(s)}$ by adding the probability of the discarded scenario $\pi^{\left(s^{*}\right)}$, see equation (4). Then, the total number of scenarios changes (e.g. $n_{s}=n_{s}-1$ ) and the distance matrix is calculated once more. This iterative process continues until certain number of scenarios is deleted (Figure 4). Finally, the reduced set of forecast errors is added to a specific wind power forecast (Figure 5). In the context of Stochastic Unit Commitment little discussion is offered regarding the right number of survival scenarios (after applying reduction techniques) that adequately represent a random parameter (e.g. wind power forecast error) due to most authors rely on the maximum computational feasible number of scenarios. A common practice is to construct the scenario tree based on the tractability of the UC solution. Then, the representation of the random parameter is tailored for that specific structure. Similar situation occurs in Scenario-based UC, in which the number of scenarios is selected based on the manageability of the UC problem rather than the appropriate representation of the random parameter. Instead, this paper follows the stability criteria proposed by K. Høyland and S.W. Wallace[12] and M. Kaut and S.W. Wallace[13], in which the quality of survival scenarios is measured by their performance on the decision model. Section V describes the implementation through a case study aiming to represent the UK power system.

\section{SCENARIO-BASEd UNIT COMMITMENT}

The core model of UC is based on Carrion and Arroyo [20] and Hobbs et al. [21] but extended to manage the variability of wind forecast error in a two-stage stochastic programing approach [22]. The formulation is summarized by the following equations:

$$
\begin{array}{rr}
\operatorname{Min} \sum_{i t} z_{i t} \gamma_{i}+S T_{i t}+\sum_{i t s} \pi_{s} \times\left(g_{i t s} \beta_{i}+V O L L \times l s_{t s}\right) \\
S T_{i t} \geq K_{i}\left(z_{i, t}-z_{i, t-1}\right) & \forall i, t
\end{array}
$$

The SUC problem is split in two parts according to the time that decisions are made (a First and a Second stage). The here and now variables in the objective function (5), such as the binary variable $Z_{i t}$ is used to determine which generators are synchronized or off-line, as well as to determine the cost of synchronizing each generator $\left(S T_{i t}\right)$ [21]. Once the First stage variables are determined, an adjustment takes place in the Second in order to accommodate deviations from forecasted quantities via available generation dispatch, $g_{\text {its. }}$. The constraints of the second stage are scenario-dependent, therefore in every scenario the power balance (7) must be met. This constraint, allows recourse variables (e.g. wind curtailment $w c_{t s}$ and load shedding $\left.l s_{t s}\right)$ to be used in order to reconcile the second stage so as to choose optimal first stage variables[22]. In this formulation, wind forecast errors are explicitly modeled in the scenario set of wind power $W_{t s}$ and weighted by their respective probability of occurrence $\pi_{s}$ in accordance with Section III.

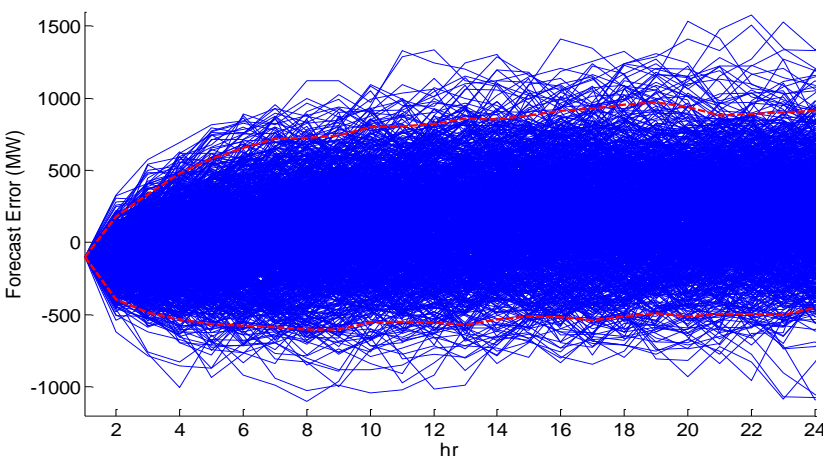

Fig. 3. 1000 Scenarios of wind power forecast error for 1 Day Ahead.

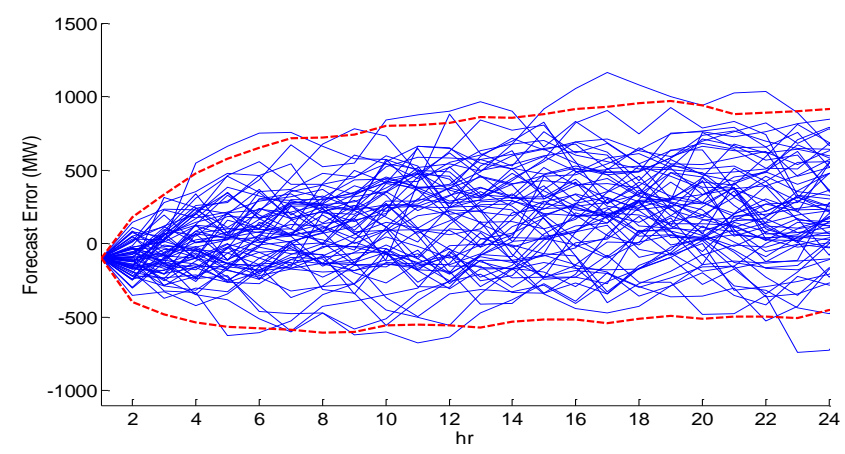

Fig. 4. 50 Scenarios of wind power forecast error after backward deletion for 1 Day Ahead.

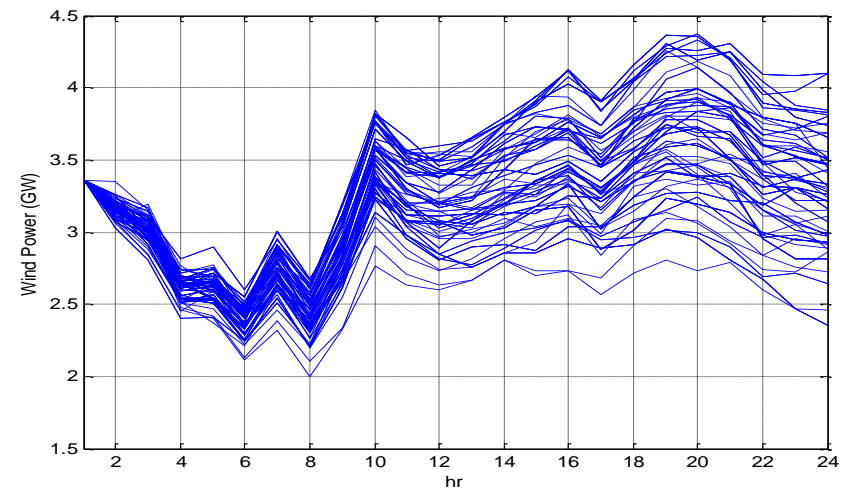

Fig. 5. 50 Scenarios of wind power for 1 Day Ahead.

$$
\begin{array}{cc}
\sum_{i} g_{i t s}+l s_{t s}-w c_{t s}=D_{t}-W_{t s} & \forall t, s \\
0 \leq l s_{t s} \leq D_{t} & \forall t, s \\
0 \leq w c_{t s} \leq W_{t} & \forall t, s \\
\sum_{i} r_{i t s} \geq S R_{t} & \forall t, s \\
\sum_{i} r d w n_{i t s} \geq S R d w n_{t} & \forall t, s \\
g_{i t s}+r_{i t s} \leq z_{i t} M x L_{i} & \forall i, t, s \\
g_{i t s}-r d w n_{i t s} \geq z_{i t} M n L_{i} & \forall i, t, s \\
r_{i t s} \leq z_{i t} M x s p_{i} & \forall i, t, s \\
r d w n_{i t s} \leq z_{i t} M x s p d_{i} & \forall i, t, s
\end{array}
$$




$$
\begin{aligned}
& g_{i t s}-g_{i, t-1, s} \leq z_{i, t-1} M x \operatorname{Inc} c_{i}+U R_{i}\left(z_{i t}-z_{i, t-1}\right) \\
& +M x L_{i}\left(1-z_{i t}\right) \\
& \forall i, s, \forall t=2 \ldots T \\
& g_{i, t-1, s}-g_{i t s} \leq z_{i t} M x D e c_{i}+D R_{i}\left(z_{i, t-1}-z_{i t}\right) \\
& +M x L_{i}\left(1-z_{i, t-1}\right) \\
& \forall i, s, \forall t=2 \ldots T \\
& \sum_{n>t}^{t+U T_{i}-1} z_{\text {in }} \geq\left(z_{i t}-z_{i, t-1}\right) \\
& \forall i, \forall t=1 \ldots T-U T_{i}+1 \\
& \sum_{n>t}^{t+D T_{i}-1}\left(1-z_{i n}\right) \geq\left(z_{i, t-1}-z_{i t}\right) \\
& \forall i, \forall t=1 \ldots T-U T_{i}+1
\end{aligned}
$$

In this paper, the reserve provided is in the tertiary regulation interval [23]; and it is itemized into the following main components: Contingency reserves, spare capacity to provide frequency regulation and reserve to accommodate wind power deviations. The first two items are accounted in $S R_{t}$ (10) as a percentage of the load (e.g. 6\% for contingency and $1.5 \%$ for frequency regulation services)[24]. The same rationale is used to provide downward reserve $S R d w n_{t}$ (11). Furthermore, equations $(12-15)$ are enforced to accommodate the upward and downward reserves among the generating units according to their response characteristics $\left(M x s p_{i} \& M x s p d_{i}\right)$, these parameters were estimated according to $[14,25]$. Previous set of constraints is based on Hobbs et al. [21]. Then, Stochastic UC dynamically allocates reserves through a set of scenarios that capture the variability of wind. Results and more abundant descriptions are given in Section V.B where additional generators are quantified according to different accuracy in prediction (Figure 9) and Section V.C offers a sensitivity analysis of reserves as a function of different scenario sets, Figure 10. Lastly, ramping constraints (16 - 17) ensure smooth operation of thermal fleet, avoiding abrupt changes in consecutive hours and the minimum number of hours that each generator has to remain on/off-line are implemented in equations (18) and (19).

\section{Case Study}

The case study correspond to the UK system, in which the generation mix is estimated based on information published by National Grid in the Seven Year Statement of 2011 [14]. The total capacity considered is nearly $70 \mathrm{GW}$ (approximately 265 generators), typified by low flexible (Nuclear plants, 10GW), medium flexible (Coal plants, 20GW) and high flexible (Gas plants, 30GW). Data for the cost of production per technology, capacity, ramping, min up \& down time were validated with the website of the Balancing Mechanism Reporting System [16] and available information from exploratory analyses of NG [14]. The forecast of wind generation comprises three sets that correspond to different horizon ( 7 days, 1 day and 4 hours ahead). The data base consists of twenty three months with sampling interval of 1 hour resolution, from January 2012 to November 2013 (see Section II). The Scenario-based UC described in Section IV has been coded in AIMMS[26], using CPLEX as solver, executed on Intel Core i5, with 4 GB of RAM and optimality gap of $0.1 \%$.

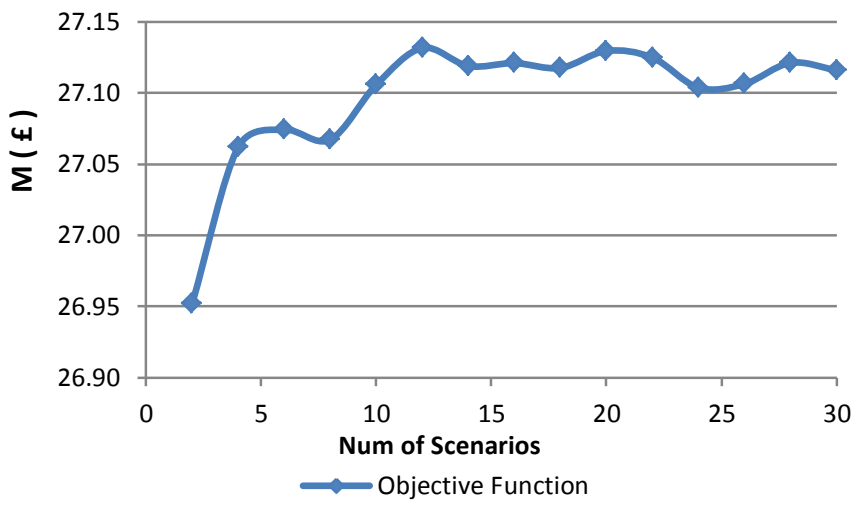

Fig. 6. Objective Function (OF) as a function of number of scenarios for one day ahead.

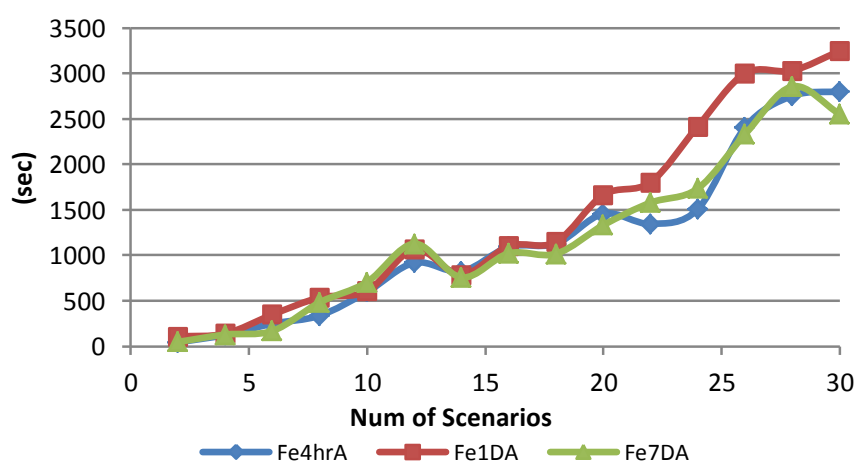

Fig. 7. Computation time of SUC for different forecast errors (4hrs, 1 day and 7 days) as a function of different Secnario Sets

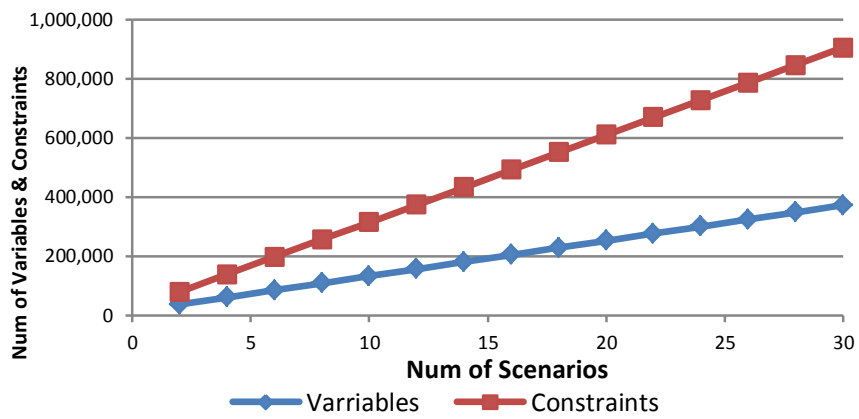

Fig. 8. Number of Variables and Constraints as a function of number of scenarios.

\section{A. Stability Requirement}

In this paper, a balance is pursued where quantity does not compromise the quality of the scenario set. Initially $\mathrm{K}$. Høyland and S.W. Wallace [12] and M. Kaut and S.W. Wallace [13] developed a framework to test limited outcomes of Moment Matching methods on portfolio optimization problems. Hence, the concept is general enough that can be extended to any scenario generation \& reduction method. The first phase consists of characterizing the random parameter (see Section II), for more sophisticated formulations regarding wind forecast error consult $[27,28]$ and the references therein. 


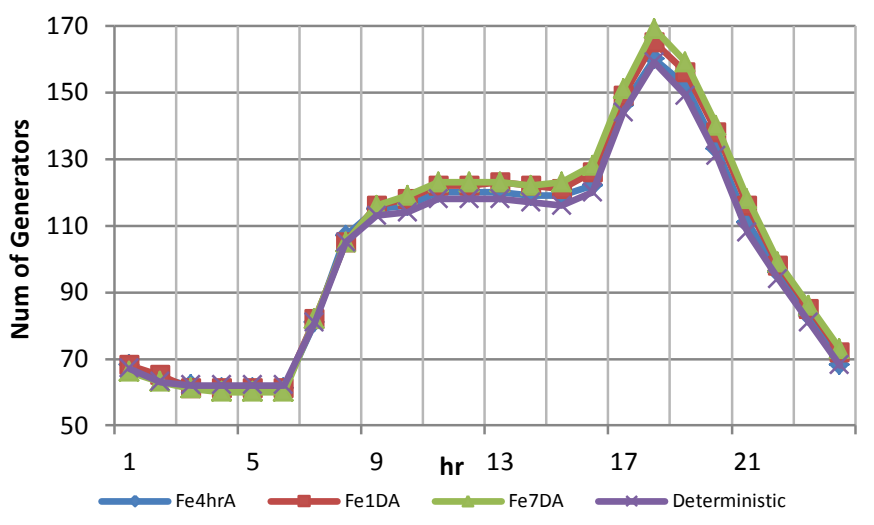

Fig. 9. Number of committed generators for deterministic and stochastic UC considering the effect of different wind forecast errors (7 days, 1 day and 4 hours ahead).

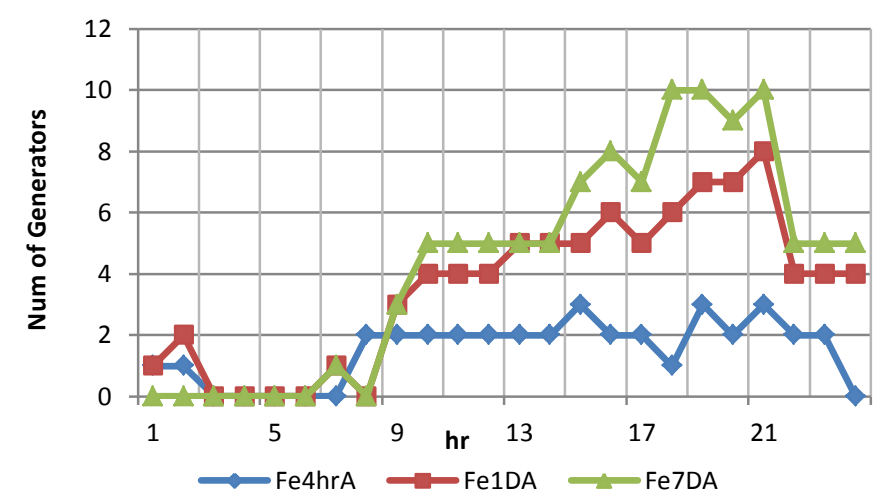

Fig. 10. Difference in the number of commited generators between Determinisctic UC and Stochastic UC considerent different wind forecast error (7 days, 1 day and 4 hours ahead).

The second phase of the proposal is to analyze the quality of survival scenario set (after applying reduction techniques) but not in terms of representing the true distribution of the random parameter. Instead, the quality is measured by the performance that the final scenario set has on the decision model. The rationale is based on the assumption that if relevant properties are embodied in the scenario generation \& reduction method, all scenarios sets provided by them should lead to the same objective function value in the decision model (SUC). Hence, Figure 6 is meant to show the stability requirement with different set of scenarios; all of them were created using same methodology (Section III). The data base comprises forecast error (1 day ahead) of nearly two years (Section II), the case study is the UK system described in Section V. Thus, Figure 6 shows certain degree of stability in the objective function after 15 scenarios, which represent a good compromise between quality, quantity and the time that is required to be solved. For this particular case, it can be seen that a reduction in computation time could be as large as two thirds of the time that it would take with a large number of scenarios (e.g. 30 scenarios). The computation time for all cases is presented in Figure 7. The cost of the objective function corresponds to the schedule of one day ahead (Tuesday, November 12th 2013). Lastly, Figure 8 shows the relationship between number of variables and constraints as a function of the increased number of scenarios.

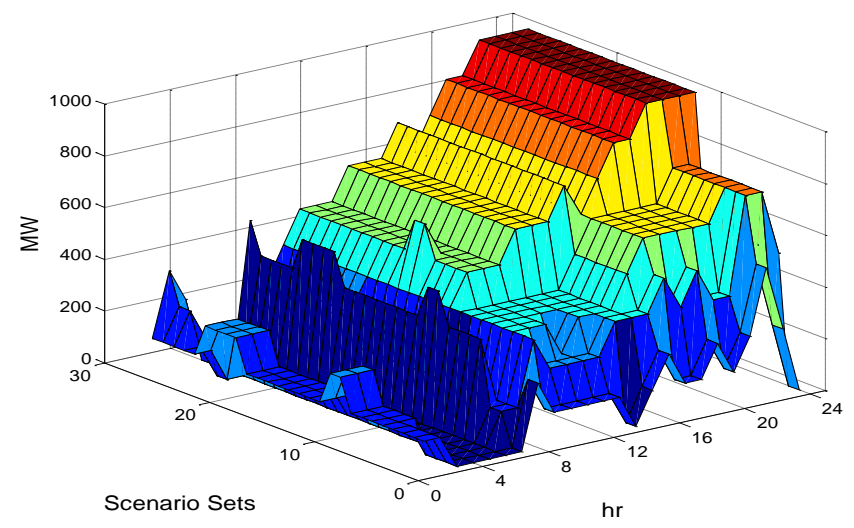

Fig. 11. Additional Reserve due to Wind Power Forecast Error as a function of different Secnario Sets in one day ahead.

TABLE I. COMPARISON OF ACCURACY FOR DIFFERENT FORECAST ERRORS

\begin{tabular}{|c|c|c|}
\hline \multirow{2}{*}{} & \multicolumn{2}{|c|}{ Improvement with respect to } \\
\cline { 2 - 3 } Fe7DA & Fe4HrA & Fe1DA \\
\hline Fe1DA & $73 \%$ & $68 \%$ \\
\hline
\end{tabular}

\section{B. Forecasting Accuracy}

The second experiment addresses the forecasting accuracy on SUC. To date various methods [3,7] affect the statistical properties (e.g. standard deviation, kurtosis and skewness) of the forecast error to diminish/improve the prediction quality. However, the data base described in Section II does not need to be shaped as the forecast that is prepared for different needs of the system ( 7 days, 1 day and 4 hours ahead) implicitly contains different degrees of accuracy. Thus, Table I compares the Mean Absolute Error (MAE) with the skill score method [29]. For instance, the forecast that is closer to real time (Fe4hrA) performs $73 \%$ better than the forecast of 7 days ahead (Fe7DA). Moreover, Figure 9 illustrates the number of committed generators considering the effect of different wind forecast errors with the SUC tool. Then, Figure 10 shows the quantification of those additional generators needed to cope with different accuracy in prediction. These values are obtained as a difference between deterministic and the stochastic solution. Hence, better forecast led to fewer generators needed to compensate for lack of accuracy.

\section{Quantification of reserves due to Wind Forecast Errors}

Next analysis is focused on reserves allocated for the SUC tool in response to wind power forecast errors, Figure 11 depicts the results. The data base for this experiment is wind forecast errors of one day ahead (Fe1DA) that is represented by different scenario sets. Then, these reserves are quantified by comparing the committed capacity of the stochastic and deterministic solution. Interestingly, a well-defined pattern is obtained after 15 scenarios, in accordance with the stability requirement of Section V.A. Furthermore, the observed trend in these reserves is similar to the forecast error (see Figure 5), which increases with the time horizon. In general, it was observed that expensive generators had more active participation to provide reserves due to higher operating cost and larger ramping characteristics. 


\section{CONCLUSIONS}

Making decisions based on the maximum number of feasible scenarios might underestimate/overestimate the random parameter that is required to be simulated in SUC problems. Therefore, a tradeoff between quantity and quality should be pursued. In this paper, the scenario generation \& reduction method were tested together with the decision model. Along the way of appraising both techniques, it was found that the representation of wind power forecast error not necessarily imply a large number of scenarios, in particular for the forecast that is closer to real time. In some cases, savings in computation time can be as much as two thirds. Additionally, it was showed that better predictions led to fewer generators needed to compensate for lack of accuracy.

\section{REFERENCES}

[1] M. A. Ortega-Vazquez and D. S. Kirschen, "Estimating the Spinning Reserve Requirements in Systems With Significant Wind Power Generation Penetration," Power Systems, IEEE Transactions on, vol. 24, pp. 114-124, 2009.

[2] J. Ma, V. Silva, R. Belhomme, D. S. Kirschen, and L. F. Ochoa, "Evaluating and Planning Flexibility in Sustainable Power Systems," Sustainable Energy, IEEE Transactions on, vol. PP, pp. 1-10, 2012.

[3] B. Hasche, R. Barth, and D. J. Swider, "Effects of improved wind forecasts on operational costs in the German electricity System," EcoMod Conference on Energy and Environmental Modeling, 2007.

[4] H. Holttinen, M. Milligan, E. Ela, N. Menemenlis, J. Dobschinski, B. Rawn, R. J. Bessa, D. Flynn, E. Gomez-Lazaro, and N. K. Detlefsen, "Methodologies to Determine Operating Reserves Due to Increased Wind Power," Sustainable Energy, IEEE Transactions on, vol. 3, pp. 713-723, 2012.

[5] "The Statement of the Energy Balancing Cost Target Modelling Methodology," National Grid, Tech. Rep.2013.

[6] R. Barth, P. Meibom, and C. Weber, "Simulation of short-term forecasts of wind and load for a stochastic scheduling model," in Power and Energy Society General Meeting, 2011 IEEE, 2011, pp. 1-8.

[7] C. Lowery and M. O'Malley, "Impact of Wind Forecast Error Statistics Upon Unit Commitment," Sustainable Energy, IEEE Transactions on, vol. 3, pp. 760-768, 2012.

[8] M. Qadrdan, W. Jianzhong, N. Jenkins, and J. Ekanayake, "Operating Strategies for a GB Integrated Gas and Electricity Network Considering the Uncertainty in Wind Power Forecasts," Sustainable Energy, IEEE Transactions on, vol. 5, pp. 128-138, 2014.

[9] N. Zhang, C. Kang, Q. Xia, and J. Liang, "Modeling Conditional Forecast Error for Wind Power in Generation Scheduling," Power Systems, IEEE Transactions on, vol. 29, pp. 1316-1324, 2014.

[10] J. M. Morales, A. J. Conejo, and J. Perez-Ruiz, "Economic Valuation of Reserves in Power Systems With High Penetration of Wind Power," Power Systems, IEEE Transactions on, vol. 24, pp. 900-910, 2009.

[11] A. Greenhall, "Wind Scenarios for Stochastic Energy Scheduling," PhD Thesis, Electrical Engineering, University of Washington, 2013.
[12] K. Høyland and S. W. Wallace, "Generating Scenario Trees for Multistage Decision Problems," Management Science, vol. 47, pp. 295$307,2001$.

[13] M. Kaut and S. W. Wallace, "Evaluation of scenario-generation methods for stochastic programming," 2003.

[14] N. Grid. National electricity transmission system: Seven year statement [Online]. Available: http://www.nationalgrid.com/uk/Electricity/SYS/current

[15] New wind power forecasting system goes on trial at National Grid [Online]. Available: http://www.nationalgrid.com/uk/Media+Centre/pressReleases/2011/25.0 5.11+wind+forecasting.htm

[16] neta - The New Electricity Trading Arrangements [Online]. Available: http://bmreports.com/

[17] A. Shortt, J. Kiviluoma, and M. O'Malley, "Accommodating Variability in Generation Planning," Power Systems, IEEE Transactions on, vol. 28, pp. 158-169, 2013.

[18] G. E. P. Box and G. M. Jenkins, Times Series Analysis: Forecasting and Control: Holden-Day, 1976.

[19] R. Barth, L. Soder, C. Weber, H. Brand, and D. Swider., "Methodology of the Scenario Tree Tool," University of Stuttgart 2006.

[20] M. Carrion and J. M. Arroyo, "A computationally efficient mixedinteger linear formulation for the thermal unit commitment problem," Power Systems, IEEE Transactions on, vol. 21, pp. 1371-1378, 2006.

[21] B. F. Hobbs, M. H. Rothkopf, R. P. O'Neill, and H.-p. Chao, The Next Generation of Electric Power Unit Commitment Models. Massachusetts: Kluwer Academic, 2001.

[22] F. Bouffard and F. D. Galiana, "Stochastic Security for Operations Planning With Significant Wind Power Generation," Power Systems, IEEE Transactions on, vol. 23, pp. 306-316, 2008.

[23] A. J. Wood and B. F. Wollenberg, Power Generation, Operation, and Control, 2nd ed. New York: Wiley, 1996.

[24] "Exploration of High-Penetration Renewable Electricity Futures," NREL2012.

[25] E. Mariani and S. S. Murthy, ADVANCED LOAD DISPATCH FOR POWER SYSTEMS: Principles, Practices and Economies. London: Springer, 1997.

[26] AIMMS Modeling Languague [Online]. Available: http://www.aimms.com

[27] M. Lange, "On the Uncertainty of Wind Power Predictions-Analysis of the Forecast Accuracy and Statistical Distribution of Errors," Journal of Solar Energy Engineering, vol. 127, pp. 177-184, 2005.

[28] B. Hodge and M. Milligan, "Wind power forecasting error distributions over multiple timescales," in Power and Energy Society General Meeting, 2011 IEEE, 2011, pp. 1-8.

[29] P. Pinson, "Estimation of the uncertainty in wind power forecasting," 2006. 